Formal assessment

\author{
Kathleen K. Smith
}

Functional Vertebrate Morphology. Edited by Milton Hildebrand, Dennis M. Bramble, Karel F. Liem and David B. Wake. Harvard University Press:1985. Pp. 544. $\$ 35, £ 29.75$.

Mriton Hildebrand and his colleagues have compiled an excellent introduction to the literature, questions and approaches of functional vertebrate morphology. The book covers a large range of topics, and most vertebrate groups are discussed. Each chapter has been written by a leading worker, and although specialists may take exception to certain aspects of each one of them, the contributors do provide clear overviews of the issues involved.

In "Functional Adaptation in Skeletal Structures", for example, Lanyon and Rubin demonstrate the remarkable plasticity of bone, while in his article Goslow discusses the difficulty in disentangling various factors important in the neural control of locomotion. Much of the variation between chapters reflects their differing scope - topics addressed range from relatively narrow ones, such as "Digging of Quadrupeds" (Hildebrand) or "Terrestrial Locomotion Without Appendages" (Edwards), to immense ones such as "The Vertebrate Eye" (Levine) or "The Octavolateralis System" (Fay and Popper). These latter contributions, quite understandably, are not completely successful in summarizing their subject matter.

Some of the authors provide good, general overviews of an area (for example, Lauder on aquatic feeding in lower vertebrates, and Bramble and Wake on feeding mechanisms of lower tetrapods), whereas others, such as "Mastication, Food Transport and Swallowing" by Hiiemae and Crompton, are largely reviews of the work of one particular research group. A typical approach is to discuss the mechanical requirements of any activity, and then summarize the means by which animals meet them. Emerson ("Jumping and Leaping") and McNeill Alexander ("Body Support, Scaling and Allometry"), for example, provide quantitative analyses, predictions about animal form and finally tests of those predictions based on comparative data.

Only selected topics can be covered in a single volume dealing with such a large field, and the book is concerned almost entirely with the musculo-skeletal system; of the 18 chapters, 11 discuss locomotion and the axial skeleton, and 3 address feeding. This may in fact reflect vertebrate functional morphology as it is practised today, but a reader expecting a general introductory text might be surprised to final chapter, "Morphology: Current Approaches and Concepts", by Liem and Wake. Here, "equilibrium analysis", which characterizes most of the contributions, is contrasted with an analysis of "intrinsic organizational features". This latter approach, which includes analyses of development, of phylogenetic history and of constraint, is currently the subject of much discussion, but receives little attention in the main portion of the text.

Functional Vertebrate Morphology is an outstanding summary of work in this field over the past 25 years and of the fruits of "equilibrium analysis". It is clear that success in the future will depend on the resolution of the differences between these two approaches, and the development of a specific and rigorous methodology for the study of the organism.

Kathleen K. Smith is an Assistant Professor in the Department of Anatomy, Duke University Medical Center, Box 3011, Durham, North Carolina 27710, USA.

\section{Back to the basics}

\section{John C. Brandt}

Physics of Comets. By K.S. Krishna Swamy. World Scientific/Wiley: 1986. Pp. 273. $£ 22, \$ 27.60$

COMET scientists are currently sorting through the bountiful harvest of data resulting from the direct observation of two comets. These comets, Halley and Giacobini-Zinner, have over the past year been probed directly, observed from rockets and spacecraft above the Earth and studied extensively by the networks of the International Halley Watch.

Thanks to these observations we now have a good idea of how the solar wind interacts with a comet to produce the large-scale plasma structures and the draped magnetic field. We now know that the spectacular disconnection events, where the entire plasma tail comes off the comet, are quite common. We have also learned that the molecular gas near the nucleus is composed primarily of water and that the principal minor constituents of this gas are carbon monoxide and carbon dioxide. The excellent images the spacecraft obtained of the nucleus reveal that it is shaped like a peanut or potato, has a very dark crust and emits material from sunward jets. More information will be available when results from a number of disciplines are brought together and compared.

Physics of Comets was completed before this recent burst of activity. The author's goal was to write a textbook that would discuss "the basic physical principles pertaining to various cometary phenomena", and the topics covered in- clude the basic radiation laws, atomic and molecular spectroscopy, light scattering theory, vaporization theory and celestial mechanics. When appropriate, the cometary observations are described and the physics applied to the phenomena. For example, after a discussion of basic spectroscopy and cometary spectra, gas production rates are derived. Problems follow each chapter and an index is included.

Some of the descriptive material is now out of date (though the basic physics remains largely relevant) and there are a few questions concerning the balance. Mie scattering theory is extensively discussed, but although the theory applies to some of the dust in comets (possibly in the tails) other particles are very porous and irregularly shaped. This is suggested by the observation of negative polarization and by the appearance of cometary particles in the upper atmosphere. The author notes the limitations, but his emphasis on Mie theory may be inappropriate. The other area of concern is plasma physics. Some description of plasma phenomena in comets is given, but the physics necessary for interpretation is not developed. Comets are a rich source of such phenomena, and an accessible cosmic laboratory, and a complete understanding of them requires an understanding of their pervasive plasma phenomena.

The author has succeeded in producing a useful text that should be acquired by libraries and serious students of comets. The main problem with the book is timing. Those interested in current progress must look elsewhere.

John C. Brandt is Chief of the Laboratory for Astronomy and Solar Physics, NASAGoddard Space Flight Center, Greenbelt, Maryland 20771 , USA. 